\title{
FINDING OPTIMAL INTEGRAL SAMPLING LATTICES FOR A GIVEN FREQUENCY SUPPORT IN MULTIDIMENSIONS
}

\author{
Yue M. Lu and Minh N. Do \\ Department of Electrical and Computer Engineering \\ University of Illinois at Urbana-Champaign, Urbana, IL 61801, USA \\ Email: $\{$ yuelu, minhdo\}@uiuc.edu; Web: www.ifp.uiuc.edu/ $\{$ yuelu, minhdo $\}$
}

\begin{abstract}
The search for alias-free sampling lattices for a given frequency support, in particular those lattices achieving minimum sampling densities, is a fundamental issue in various applications of signal and image processing. In this paper, we propose an efficient computational procedure to find all alias-free integral sampling lattices for a given frequency support with minimum sampling density. Central to this algorithm is a novel condition linking the alias-free sampling with the Fourier transform of the indicator function defined on the frequency support. We study the computation of these Fourier transforms based on the divergence theorem, and propose a simple closed-form formula for a fairly general class of support regions consisting of arbitrary $N$-dimensional polytopes, with polygons in 2-D and polyhedra in 3-D as special cases. The proposed algorithm can be useful in a variety of applications involving the design of efficient acquisition schemes for multidimensional bandlimited signals.
\end{abstract}

Index Terms - Densest sampling, critical sampling, packing, tiling, maximal decimation.

\section{INTRODUCTION}

Sampling is a cornerstone of multirate signal processing. The classical sampling theorem [1] states that a one-dimensional bandlimited signal can be exactly reconstructed from its samples if the sampling rate is beyond the Nyquist rate. The situation is similar in multidimensional cases. In general, the effect of the sampling process in the frequency domain is that the spectrum of the original signal gets replicated over a lattice whose density is inversely proportional to the sampling density. If the shifted copies of the spectrum do not overlap with the baseband, then we have an alias-free sampling; consequently, the original signal can be reconstructed from its downsampled version by applying an ideal interpolation filter supported on the baseband.

The search for alias-free sampling lattices for a given frequency support, and in particular those lattices achieving minimum sampling densities, is a fundamental issue in various applications involving the design of efficient acquisition schemes

This work was supported by the US National Science Foundation under Grant CCR-0237633 (CAREER). for bandlimited signals. While this problem can be solved in 1$\mathrm{D}$ via visual inspection, it becomes very challenging in higher dimensions. A special case of alias-free sampling, i.e., maximal decimation, is also important in the design of criticallysampled filter banks [2].

In this paper, we propose a computational procedure that, when given the Fourier transform [denoted by $\widehat{\mathbb{1}}_{\mathcal{X}}(\omega)$ ] of the indicator function defined on the frequency support region $\mathcal{X}$, can find all alias-free integral sampling lattices for $\mathcal{X}$ with the minimum density. Central to this algorithm is a novel condition linking the alias-free sampling with the value of $\widehat{\mathbb{1}}_{\mathcal{X}}(\boldsymbol{\omega})$ at integral points. To systematically investigate all possible sampling geometry, we describe and employ the Hermite normal form, which provides a complete characterization for all integral lattices. Since the usefulness of the proposed algorithm depends on whether we can calculate $\widehat{\mathbb{1}}_{\mathcal{X}}(\boldsymbol{\omega})$, we study the computation of this function based on the Gauss divergence theorem. In particular, we provide a simple closed-form formula for a fairly general class of support regions consisting of arbitrary $N$-dimensional polytopes, with polygons in 2-D and polyhedra in 3-D as special cases.

The rest of the paper is organized as follows. In Section 2, we first briefly review some relevant concepts in the sampling of bandlimited signals, and then state precisely the problems we want to address in this work. We propose in Section 3 a novel condition linking the alias-free sampling (as well as maximal decimation) with the Fourier transform of the indicator function defined on the given frequency support. Section 4 presents a computational algorithm that can efficiently determine all suitable integral sampling lattices for a given frequency support at the minimum sampling density. We conclude the paper in Section 5. Due to space limitations, we only present the proofs for the most important results in this paper, and leave the proofs for all other results to [3].

Notations: Throughout the paper, $N$ represents the dimension of the signals. We use $|\boldsymbol{M}|$ to represent the absolute value of the determinant of a matrix $\boldsymbol{M}$. For a function $f(\boldsymbol{t})$, its Fourier transform is written as $\widehat{f}(\boldsymbol{\omega})$. Calligraphic letters, such as $\mathcal{X}$, represent open domains in the $N$-dimensional $(N$-D) space, with $m(\mathcal{X})$ being the Lesbegue measure (volume) of $\mathcal{X}$. Finally, $\mathbb{1}_{\mathcal{X}}(\boldsymbol{t})$ represents the indicator function defined on $\mathcal{X}$, i.e., $\mathbb{1}_{\mathcal{X}}(\boldsymbol{t})=1$ if $\boldsymbol{t} \in \mathcal{X}$ and $\mathbb{1}_{\mathcal{X}}(\boldsymbol{t})=0$ otherwise. 


\section{BACKGROUND AND PROBLEM STATEMENT}

\subsection{Sampling on Lattices}

In multidimensional multirate signal processing, the sampling operations are defined on lattices, each of which is represented by an $N \times N$ nonsingular matrix $\boldsymbol{M}$ as

$$
\operatorname{LAT}(\boldsymbol{M})=\left\{\boldsymbol{M n}: \boldsymbol{n} \in \mathbb{Z}^{N}\right\} .
$$

In this paper, we focus on discrete-time signals, and hence we further require that the sampling matrix $\boldsymbol{M}$ always be integervalued. However, most of the results presented here apply to the sampling of continuous-time signals as well [3].

For an $\boldsymbol{M}$-fold sampling, the input $x[\boldsymbol{n}]$ and the output $y[\boldsymbol{n}] \stackrel{\text { def }}{=} x[\boldsymbol{M n}]$ are related in the Fourier domain as [4]

$$
\mathcal{Y}=\boldsymbol{M}^{T} \bigcup_{\boldsymbol{n} \in \mathbb{Z}^{N}}\left(\mathcal{X}+2 \pi \boldsymbol{M}^{-T} \boldsymbol{n}\right) .
$$

where $\mathcal{X}$ is the frequency-domain support of the input signal $x[\boldsymbol{n}]$ within $(-\pi, \pi]^{N}$, and $\mathcal{Y}$ is the frequency domain support of $y[\boldsymbol{n}]$.

It follows from (1) that the frequency support $\mathcal{Y}$ of the downsampled signal can be obtained by first taking the union of all shifted copies (i.e. alias components) of $\mathcal{X}$, whose origins are moved to points on the lattice $\operatorname{LAT}\left(2 \pi \boldsymbol{M}^{-T}\right)$, and then applying a linear mapping $M^{T}$.

Definition 1 We say a frequency support $\mathcal{X} \subseteq(-\pi, \pi]^{N}$ allows an alias-free $M$-fold sampling, if different shifted copies of $\mathcal{X}$ in (1) are disjoint, i.e., for all $\boldsymbol{n}_{1} \neq \boldsymbol{n}_{2} \in \mathbb{Z}^{N}$,

$$
\left(\mathcal{X}+2 \pi \boldsymbol{M}^{-T} \boldsymbol{n}_{1}\right) \cap\left(\mathcal{X}+2 \pi \boldsymbol{M}^{-T} \boldsymbol{n}_{2}\right)=\emptyset .
$$

Furthermore, we say $\mathcal{X}$ can be maximally decimated by $\boldsymbol{M}$, if aside from the alias-free condition in (2), the union of the shifted copies also covers the entire spectrum, i.e.,

$$
\bigcup_{\boldsymbol{n} \in \mathbb{Z}^{N}}\left(\mathcal{X}+2 \pi \boldsymbol{M}^{-T} \boldsymbol{n}\right)=\mathbb{R}^{N}, \quad \text { up to a set of measure zero. }
$$

\subsection{Problem Statement}

The sampling density of a matrix $M$ is defined as $\rho_{M}=1 /|M|$, which is the number of samples retained per unit volume. It is well-known [4] that the lower bound of sampling density for alias-free sampling is $m(\mathcal{X}) /(2 \pi)^{N}$, where $m(\mathcal{X})$ is the volume of the support region $\mathcal{X}$; meanwhile, this bound is achieved when $\mathcal{X}$ can be maximally decimated. Therefore, the first problem we want to address in this paper is as follows.

Problem 1: Given a frequency support $\mathcal{X}$, can it be maximally decimated by an integral matrix? And if yes, what are the corresponding sampling matrices?

Apparently not all frequency support shapes can be maximally decimated (e.g. consider a disc-shaped region in 2-D). Hence, the second and more general problem is:

Problem 2: Given a frequency support $\mathcal{X}$, what is the achievable minimum sampling density for alias-free sampling by using integral matrices? How do we efficiently find all integral matrices achieving this minimum density?

\section{STUDY OF ALIAS-FREE SAMPLING USING FOURIER TECHNIQUES}

In this section, we study the problems of alias-free sampling and maximal decimation based on Fourier analysis. The key observation is a link between the alias-free sampling condition with the Fourier transform of the indicator function $\mathbb{1}_{\mathcal{X}}(\boldsymbol{t}) \mathrm{de}$ fined on the support $\mathcal{X}$.

For a given support $\mathcal{X}$, consider the autocorrelation function $R_{\mathcal{X}}(\boldsymbol{t})=\int \mathbb{1}_{\mathcal{X}}(\boldsymbol{u}) \mathbb{1}_{\mathcal{X}}^{*}(\boldsymbol{u}-\boldsymbol{t}) d \boldsymbol{u}$ and define a quantity

$$
O_{\mathcal{X}, \boldsymbol{M}} \stackrel{\text { def }}{=} \sum_{\boldsymbol{n} \neq \mathbf{0}} R_{\mathcal{X}}\left(2 \pi \boldsymbol{M}^{-T} \boldsymbol{n}\right)
$$

which can be interpreted as the total volume of overlapping regions between the original support $\mathcal{X}$ and all its aliasing components.

Lemma 1 A frequency region $\mathcal{X}$ allows an $M$-fold alias-free sampling, if and only if $O_{\mathcal{X}, M}=0$.

Proof: By construction, $R_{\mathcal{X}}(\boldsymbol{t}) \geq 0$ for all $\boldsymbol{t}$; thus $O_{\mathcal{X}, M}=0$ if and only if $R_{\mathcal{X}}\left(2 \pi \boldsymbol{M}^{-T} \boldsymbol{n}\right)=0$ for all $\boldsymbol{n} \in \mathbb{Z}^{N} \backslash\{\mathbf{0}\}$, which is equivalent to the alias-free condition given in (2).

Theorem 1 A frequency region $\mathcal{X}$ allows an $M$-fold alias-free sampling, if and only if

$$
\sum_{\boldsymbol{n} \in \mathbb{Z}^{N}}\left|\widehat{\mathbb{1}}_{\mathcal{X}}(\boldsymbol{M} \boldsymbol{n})\right|^{2} \leq \frac{(2 \pi)^{N}}{|\boldsymbol{M}|} m(\mathcal{X}) .
$$

Proof: From the definition of $R_{\mathcal{X}}(\boldsymbol{t})$, its Fourier transform is $\widehat{R}_{\mathcal{X}}(\boldsymbol{\omega})=\left|\widehat{\mathbb{1}}_{\mathcal{X}}(\boldsymbol{\omega})\right|^{2}$. Applying the Poisson summation formula, we have

$$
\begin{aligned}
\sum_{\boldsymbol{n} \in \mathbb{Z}^{N}} R_{\mathcal{X}}\left(2 \pi \boldsymbol{M}^{-T} \boldsymbol{n}\right) & =\frac{|\boldsymbol{M}|}{(2 \pi)^{N}} \sum_{\boldsymbol{n} \in \mathbb{Z}^{N}} \widehat{R}_{\mathcal{X}}(\boldsymbol{M} \boldsymbol{n}) \\
& =\frac{|\boldsymbol{M}|}{(2 \pi)^{N}} \sum_{\boldsymbol{n} \in \mathbb{Z}^{N}}\left|\widehat{\mathbb{1}}_{\mathcal{X}}(\boldsymbol{M} \boldsymbol{n})\right|^{2} .
\end{aligned}
$$

The overlapping term $O_{\mathcal{X}, M}$ can then be calculated as

$$
\begin{aligned}
O_{\mathcal{X}, \boldsymbol{M}} & =\sum_{\boldsymbol{n} \in \mathbb{Z}^{N}} R_{\mathcal{X}}\left(2 \pi \boldsymbol{M}^{-T} \boldsymbol{n}\right)-R_{\mathcal{X}}(\mathbf{0}) \\
& =\frac{|\boldsymbol{M}|}{(2 \pi)^{N}} \sum_{\boldsymbol{n} \in \mathbb{Z}^{N}}\left|\widehat{\mathbb{1}}_{\mathcal{X}}(\boldsymbol{M} \boldsymbol{n})\right|^{2}-m(\mathcal{X}),
\end{aligned}
$$

where in reaching (5) we have also used the fact that $R_{\mathcal{X}}(\mathbf{0})=$ $m(\mathcal{X})$. It follows from (5) that the inequality (4) is equivalent to having $O_{\mathcal{X}, M} \leq 0$. On the other hand, we always have $O_{\mathcal{X}, M} \geq 0$ from definition. Therefore, $O_{\mathcal{X}, M}=0$ and we can then apply Lemma 1.

Corollary 1 Suppose $M$ is an alias-free sampling matrix for $\mathcal{X}$, then the sampling density $\rho_{M}$ satisfies

$$
\rho_{\boldsymbol{M}} \stackrel{\text { def }}{=} \frac{1}{|\boldsymbol{M}|} \geq \frac{m(\mathcal{X})}{(2 \pi)^{N}} .
$$

The above lower bound is achieved if and only if $\mathcal{X}$ is maximally decimated by $M$. 


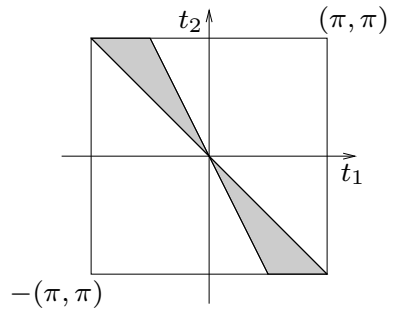

(a)

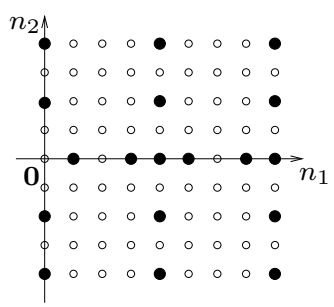

(b)
Fig. 1. (a) The ideal frequency domain support of one of the subbands from a 3-level directional filter bank. (b) The values of $\widehat{\mathbb{1}}_{\mathcal{X}}(\boldsymbol{\omega})$ at integer points. Black dots represent zero values; white dots represent nonzero values.

The above lower bound on sampling density is a well-known result in multirate signal processing [4], but is usually proved by geometric arguments. By combining the results of Theorem 1 and Corollary 1, we can derive a simpler set of conditions for the special case of maximal decimation as follows, which was originally proposed in [5].

Theorem 2 A frequency support $\mathcal{X}$ can be maximally decimated by a matrix $M$ if and only if

$\widehat{\mathbb{1}}_{\mathcal{X}}(\mathbf{0})=\frac{(2 \pi)^{N}}{|\boldsymbol{M}|}$ and $\widehat{\mathbb{1}}_{\mathcal{X}}(\boldsymbol{M n})=0$ for all $\boldsymbol{n} \in \mathbb{Z}^{N} \backslash\{\mathbf{0}\}$.

Example 1 Figure 1(a) shows the ideal wedge-shaped frequency support $\mathcal{X}$ of one directional filter bank [2] subband. Illustrated in Figure $1(b)$ are the values of $\widehat{\mathbb{1}}_{\mathcal{X}}\left(n_{1}, n_{2}\right)$ at integer points for $0 \leq n_{1} \leq 8$ and $-4 \leq n_{2} \leq 4$, with black dots representing the locations where $\widehat{\mathbb{1}}_{\mathcal{X}}\left(n_{1}, n_{2}\right)=0$. We can observe that the zero set contains a lattice generated by the diagonal matrix $\boldsymbol{M}=\operatorname{diag}(4,2)$, and hence $\boldsymbol{M}$ is a suitable candidate for maximal decimation. Actually, we can use the expression for $\widehat{\mathbb{1}}_{\mathcal{X}}(\boldsymbol{\omega})$ to show that $\boldsymbol{M}$ indeed satisfies both conditions in (7). This matches the result previously obtained from the construction of the filter bank [2].

\section{FINDING MINIMUM SAMPLING LATTICES}

Based on the result of Theorem 1, we propose in this section a computational procedure that can efficiently determine the minimum sampling lattices for a given frequency support.

\subsection{Characterization of Integral Lattices}

Given a frequency support $\mathcal{X}$, we can first calculate its volume. We know from Corollary 1 that the integral matrix $M$ for alias-free sampling must satisfy $\left.|\boldsymbol{M}| \leq \mid \frac{(2 \pi)^{N}}{m(\mathcal{X})}\right\rfloor$, where $\lfloor\alpha\rfloor$ is the largest integer smaller than or equal to a real number $\alpha$. Although there is an infinite number of integral matrices satisfying this condition, we only need to check a finite number of them, as ensured by the following result:
Proposition 1 A matrix $M$ is an alias-free sampling matrix for a frequency support $\mathcal{X}$, if and only if $\boldsymbol{M U}$ is an alias-free sampling matrix for $\mathcal{X}$, where $\boldsymbol{U}$ is an arbitrary unimodular matrix (i.e. integral matrix with $|\boldsymbol{U}|=1$ ). The same is true for maximal decimation.

For two integral matrices $\boldsymbol{M}$ and $\boldsymbol{P}$, the relation $\boldsymbol{M} \sim \boldsymbol{P}$, defined as $\boldsymbol{P}=\boldsymbol{M U}$ for some unimodular matrix $\boldsymbol{U}$, is an equivalence relation. Therefore, the corresponding equivalence classes form a partition of the set of all integral matrices whose determinants have the same absolute value. Proposition $1 \mathrm{im}$ plies that, for each equivalent class, we just need to pick a representative matrix from that class, and see if it forms an aliasfree sampling (or maximal decimation). The result then carries over to all members in the equivalent class. The following result [6] provides a complete characterization for all representative matrices.

Theorem 3 (Hermite normal form) For every integral matrix $\boldsymbol{M}$, there is a unique integral matrix $\boldsymbol{H}$, such that $\boldsymbol{M} \sim \boldsymbol{H}$ and $\boldsymbol{H}=\left[h_{i, j}\right]_{1 \leq i, j \leq N}$ is in the Hermite normal form, i.e.,

\section{1. $\boldsymbol{H}$ is upper triangular;}

$$
\text { 2. } h_{i, i} \geq 0 \text { and } 0 \leq h_{i, j}<h_{i, i} \quad(j>i ; 1 \leq i \leq N) \text {. }
$$

From definition, it is straightforward to construct all Hermite normal matrices with a given determinant. For example, when $N=2$ and the determinant is equal to 3 , there are a total of four such matrices:

$$
\left(\begin{array}{ll}
1 & 0 \\
0 & 3
\end{array}\right),\left(\begin{array}{ll}
3 & 0 \\
0 & 1
\end{array}\right),\left(\begin{array}{ll}
3 & 1 \\
0 & 1
\end{array}\right) \text {, and }\left(\begin{array}{ll}
3 & 2 \\
0 & 1
\end{array}\right) \text {. }
$$

In general, we can show [3] that, for a given determinant $\delta$, the total number of Hermite normal matrices with that determinant is less than $22 \delta^{N-1+0.001}$. This implies that, in lower dimensions cases (e.g. $N=2,3$ ) and when $\delta$ is small, we just need to check a relatively small number of candidate sampling matrices.

\subsection{The Fourier Transform of Indicator Functions}

The usefulness of condition (4) proposed in Theorem 1 depends on whether we can calculate $\widehat{\mathbb{1}}_{\mathcal{X}}(\boldsymbol{\omega})$. In general, this has to be decided on a case by case basis, according to the shape of $\mathcal{X}$. However, for a fairly large of class of support shapes consisting of $N$-D polytopes, we can have a simple closed-form formula for $\widehat{\mathbb{1}}_{\mathcal{X}}(\boldsymbol{\omega})$.

Consider a vector-valued function $F_{\boldsymbol{\omega}}(\boldsymbol{t})=\frac{j \boldsymbol{\omega}}{\|\boldsymbol{\omega}\|^{2}} e^{-j \boldsymbol{\omega} \cdot \boldsymbol{t}}$, where $\boldsymbol{\omega} \neq \mathbf{0}$ is a fixed parameter. It is easy to verify that $\operatorname{div} F_{\boldsymbol{\omega}}(\boldsymbol{t})=e^{-j \boldsymbol{\omega} \cdot \boldsymbol{t}}$, where $\operatorname{div} F_{\boldsymbol{\omega}}(\boldsymbol{t})$ is the divergence of the function. Applying the Gauss divergence theorem, we have

$$
\begin{aligned}
\widehat{\mathbb{1}}_{\mathcal{X}}(\boldsymbol{\omega}) & \stackrel{\text { def }}{=} \int_{\mathcal{X}} e^{-j \boldsymbol{\omega} \cdot \boldsymbol{t}} d \boldsymbol{t}=\int_{\mathcal{X}} \operatorname{div} F_{\boldsymbol{\omega}}(\boldsymbol{t}) d \boldsymbol{t} \\
& =\frac{j}{\|\boldsymbol{\omega}\|^{2}} \int_{\partial \mathcal{X}} e^{j \boldsymbol{\omega} \cdot \boldsymbol{t}}\left(\boldsymbol{\omega} \cdot \boldsymbol{n}_{\boldsymbol{t}}\right) d S_{\boldsymbol{t}},
\end{aligned}
$$


for any $\boldsymbol{\omega} \neq \mathbf{0}$, where $\partial \mathcal{X}$ is the boundary of $\mathcal{X}$ oriented by outward-pointing unit normal vectors $\boldsymbol{n}_{\boldsymbol{t}}$.

If $\mathcal{X}$ is a polygon in 2-D, then $\partial \mathcal{X}$ is made of a finite number of line segments. By using (8), we can simplify the computation of $\widehat{\mathbb{1}}_{\mathcal{X}}(\boldsymbol{\omega})$ from a volume integration to a finite number of 1-D line integrations, which allow for a simple closed-form expression as follows:

Theorem 4 Suppose $\mathcal{X}$ is a 2-D polygon with $K$ sides. For any $\boldsymbol{\omega} \neq \mathbf{0}$,

$$
\widehat{\mathbb{1}}_{\mathcal{X}}(\boldsymbol{\omega})=\frac{1}{\|\boldsymbol{\omega}\|^{2}} \sum_{k=1}^{K} \frac{\boldsymbol{\omega} \cdot \boldsymbol{n}_{k}}{\boldsymbol{\omega} \cdot \boldsymbol{\theta}_{k}}\left(e^{-j \boldsymbol{\omega} \cdot \boldsymbol{p}_{k}}-e^{-j \boldsymbol{\omega} \cdot \boldsymbol{q}_{k}}\right),
$$

where $\boldsymbol{p}_{k}$ and $\boldsymbol{q}_{k}$ are the two vertices of the kth side; $\boldsymbol{\theta}_{k}=$ $\frac{\boldsymbol{q}_{k}-\boldsymbol{p}_{k}}{\left\|\boldsymbol{q}_{k}-\boldsymbol{p}_{k}\right\|}$ is the unit tangent vector pointing from $\boldsymbol{p}_{k}$ to $\boldsymbol{q}_{k}$; and $\boldsymbol{n}_{k}$ is the outward-pointing unit normal vector of the kth side.

The higher dimensional cases for polyhedra and polytopes can be done in a similar fashion, and we leave the details to [3].

\subsection{Algorithm}

For a given frequency support region $\mathcal{X}$, if $\widehat{\mathbb{1}}_{\mathcal{X}}(\boldsymbol{\omega})$ can be computed, we can then use the following computational procedure to determine the minimum alias-free sampling lattices for $\mathcal{X}$.

1. Compute $\frac{(2 \pi)^{N}}{m(\mathcal{X})}$, and start with the largest possible determinant, i.e., set $d=\left\lfloor\frac{(2 \pi)^{N}}{m(\mathcal{X})}\right\rfloor$.

2. From the definition given in Theorem 3, construct a list of all Hermite normal matrices with determinants $d$.

3. For each matrix $M$ in the above list, check

$$
\sum_{\boldsymbol{n} \in \mathbb{Z}^{N},\|\boldsymbol{n}\| \leq L}\left|\widehat{\mathbb{1}}_{\mathcal{X}}(\boldsymbol{M n})\right|^{2} \leq \frac{(2 \pi)^{N}}{|\boldsymbol{M}|} m(\mathcal{X}),
$$

where $L$ is a predetermined number defining the range of computation.

4. If (9) is not satisfied by any matrix in the list, set $d=$ $d-1$ and go back to Step 2 .

5. Otherwise, present all the matrices in the current list satisfying (9). Furthermore, if the current value of $d$ is equal to $\frac{(2 \pi)^{N}}{m(\mathcal{X})}$, then we have maximal decimation.

Note that we can only compute a finite sum in (9), instead of the infinite sum as required in (4). Therefore, (9) is only a necessary condition for $M$ to be an alias-free sampling matrix of $\mathcal{X}$. However, by appropriately choosing the search range $L$, we can make sure that the overlapping term $O_{\mathcal{X}, M}$ [see (3)] for the reported matrices, if not exactly zero, is still less than an arbitrarily small amount. We leave the details of how to choose $L$ to [3].

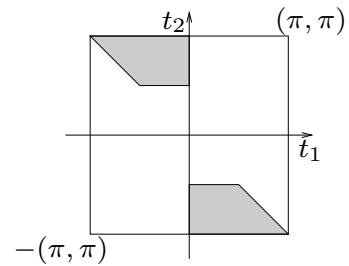

Fig. 2. The ideal frequency support of one contourlet subband.

Example 2 Figure 2 shows the ideal frequency domain support of one directional multiresolution subband from the contourlet transform [7]. Since $(2 \pi)^{2} / m(\mathcal{X})=16 / 3 \approx 5.33$, we know from Corollary 1 that $\mathcal{X}$ cannot be maximally decimated by any integral matrices. Meanwhile, for any alias-free sampling matrix $\boldsymbol{M}$ for $\mathcal{X}$, we must have $|\boldsymbol{M}| \leq 5$.

Since $\mathcal{X}$ is a polygonal region, we can easily calculate $\widehat{\mathbb{1}}_{\mathcal{X}}(\boldsymbol{\omega})$ from Theorem 4. Applying the algorithm proposed above, we find the achievable minimum sampling density for $\mathcal{X}$ is $1 / 4$, obtained by the following matrices

$$
\left(\begin{array}{ll}
1 & 0 \\
0 & 4
\end{array}\right),\left(\begin{array}{ll}
2 & 0 \\
0 & 2
\end{array}\right),\left(\begin{array}{ll}
4 & 1 \\
0 & 1
\end{array}\right) \text {, and }\left(\begin{array}{ll}
4 & 2 \\
0 & 1
\end{array}\right) \text {, }
$$

which represent four distinctive lattices.

\section{CONCLUSION}

In this paper, we proposed a novel condition linking the aliasfree sampling of a given frequency region with the Fourier transform of the indicator function defined on that region. For cases when the above Fourier transform can be computed, we propose an efficient algorithm to find all integral lattices that can sample the given frequency support with minimum sampling density. We envision that the proposed condition and computational procedure can be used in a wide range of applications involving the design of efficient acquisition schemes for multidimensional bandlimited signals.

\section{REFERENCES}

[1] A. Jerri, "The Shannon sampling theorem - Its various extensions and applications: A tutorial review," Proc. IEEE, vol. 65, pp. 1565-1596, 1977.

[2] R. H. Bamberger and M. J. T. Smith, "A filter bank for the directional decomposition of images: theory and design," IEEE Trans. Signal Proc., vol. 40, no. 4, pp. 882-893, April 1992.

[3] Y. M. Lu, M. N. Do, and R. S. Laugesen, "A computable Fourier condition generating alias-free sampling lattices," 2007, to be submitted.

[4] P. P. Vaidyanathan, Multirate Systems and Filter Banks, Prentice Hall, 1993.

[5] M. N. Kolountzakis and J. C. Lagarias, "Tilings of the line by translates of a function," Duke Math. J., vol. 82, no. 3, pp. 653678, 1996.

[6] M. Newman, Integral Matrices, Academic Press, 1972.

[7] M. N. Do and M. Vetterli, "The contourlet transform: An efficient directional multiresolution image representation," IEEE Trans. Image Proc., vol. 14, no. 12, pp. 2091-2106, December 2005. 